\title{
A randomised controlled trial of nasal continuous positive airway pressure on insulin sensitivity in obstructive sleep apnoea
}

\author{
J.C.M. Lam*, B. Lam*, T.J. Yao\#, A.Y.K. Lai*, C.G. Ooi ${ }^{\mp}$, S. Tam ${ }^{+}$, \\ K.S.L. Lam ${ }^{\star, \$}$ and M.S.M. Ip*,\$
}

ABSTRACT: The effects of treatment of obstructive sleep apnoea (OSA) on glucose metabolism have been investigated previously with conflicting results. This study evaluated the impact of nasal continuous positive airway pressure (nCPAP) treatment of OSA on insulin sensitivity.

Males with moderate/severe OSA and no significant comorbidity were randomised to a therapeutic or sham nCPAP treatment group for 1 week and then reassessed. Those who received therapeutic nCPAP were further evaluated at 12 weeks. Insulin sensitivity (Kitt) was estimated by the short insulin tolerance test. Other evaluations included blood pressure, metabolic profile, urinary catecholamines and intra-abdominal fat.

In total, 61 Chinese subjects were randomised. 31 subjects receiving therapeutic nCPAP showed an increase in Kitt $\left(6.6 \pm 2.9\right.$ to $\left.7.6 \pm 3.2 \% \cdot \mathrm{min}^{-1} ; \mathrm{p}=0.017\right)$, while the 30 patients on sham CPAP had no significant change, and the changes in Kitt were different between the two groups $(p=0.022)$. At 12 weeks, improvement in $\mathrm{Kitt}$ was seen in 20 subjects with $\mathrm{BMI} \geqslant 25 \mathrm{~kg} \cdot \mathrm{m}^{-2}$ (median (interquartile range) $28.3(26.6-31.5) ; p=0.044)$, but not in the nine subjects with $\mathrm{BMI}<25 \mathrm{~kg} \cdot \mathrm{m}^{-2}$, or the entire group.

The findings indicate that therapeutic nCPAP treatment of OSA for 1 week improved insulin sensitivity in nondiabetic males, and the improvement appeared to be maintained after 12 weeks of treatment in those with moderate obesity.

KEYWORDS: Insulin sensitivity, nasal continuous positive airway pressure, obstructive sleep apnoea, randomised controlled trial

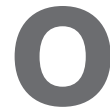

bstructive sleep apnoea (OSA) is associated with increased cardiovascular risk [1-3], and its prevalence is closely linked to the worldwide obesity epidemic [4]. Obesity is not only a major risk factor for sleep apnoea but also for cardiometabolic diseases. Insulin resistance/impaired glucose tolerance is strongly associated with visceral adiposity, which may play a role in the underlying pathophysiological pathways to altered glucose metabolism [5].

A number of studies have reported impaired glucose metabolism in OSA patients, independent of obesity [6]. However, a 4-yr follow-up of a population cohort failed to find increased incidence of diabetes in those with baseline OSA [7]. Studies on the effects of nasal continuous positive airway pressure (nCPAP) treatment on insulin resistance/diabetes in subjects with OSA have reported conflicting results [6, 8-17]. Many of these studies were limited by their variable use of control groups, small sample sizes and inadequate control of confounding factors.

We hypothesise that OSA may impair insulin sensitivity, which can be reverted by effective treatment of OSA when diabetes has not set in. Thus, this study was designed to investigate the effects of nCPAP treatment of OSA on insulin sensitivity in males without overt diabetes mellitus.

\section{METHODS AND MATERIALS}

\section{Patient recruitment}

Chinese males aged between 21-65 yrs, who underwent overnight diagnostic polysomnography (PSG) at the Sleep Laboratory (Queen Mary

\section{AFFILIATIONS}

*Depts of Medicine

'Radiology, Queen Mary Hospital,

The University of Hong Kong,

\#Medical Statistics Unit, Clinical

Trial Centre, The University of Hong Kong,

${ }^{\S}$ Research Centre for Heart, Brain, Hormone and Healthy Aging, The University of Hong Kong, and ${ }^{+}$Dept of Clinical Biochemistry, Queen Mary Hospital, Hong Kong, SAR, China.

\section{CORRESPONDENCE}

M.S.M. Ip

University Dept of Medicine

Queen Mary Hospital

102 Pokfulam Road

Hong Kong

SAR

China

E-mail: msmip@hkucc.hku.hk

Received:

March 242009

Accepted after revision:

June 182009

First published online:

July 162009 
Hospital, Hong Kong) were recruited from October 2002 to June 2007 (except during the period March to September 2003 when sleep services were suspended). Some of these subjects participated in the cohort study of OSA and cardiometabolic risk [18]. Inclusion criteria were: OSA with an apnoea/ hypopnoea index $(\mathrm{AHI}) \geqslant 15$ events $\cdot \mathrm{h}^{-1}$; continuous positive airway pressure (CPAP) naïve; no significant medical history; not taking any medications; and able to give written informed consent. Exclusion criteria were: fasting glucose $\geqslant 6.1 \mathrm{mmol} \cdot \mathrm{L}^{-1}$; sleep disorders other than OSA; excessive sleepiness causing potential harm (e.g. in drivers) and necessitating immediate institution of nCPAP therapy; habitual drinker of more than three times per week; morbid obesity with a body mass index (BMI) of $\geqslant 35 \mathrm{~kg} \cdot \mathrm{m}^{-2}$.

\section{Polysomnography}

Subjects underwent an overnight 16-channel PSG (Alice 3/5 diagnostic sleep system; Respironics Inc., Youngwood, PA, USA) as previously described [18]. PSG recordings were manually scored according to standard criteria [18].

\section{CPAP machines}

Subjects receiving therapeutic nCPAP were given autotitrating machines for 1 week (Remstar Pro; Respironics Inc.). Those receiving sham (placebo) $\mathrm{CPAP}$ had the same machines but they had been modified to deliver a pressure of $0-1 \mathrm{cmH}_{2} \mathrm{O}$ according to previously used methodology $[19,20]$, with a flow restricting connector and extra holes created at the exhalation port of the mask to allow air to escape and to prevent rebreathing of $\mathrm{CO}_{2}$. The subtherapeutic pressure was confirmed with a manometer before and after the 1-week study period. Compliance data were downloaded from built-in memory cards in the nCPAP machines.

\section{Protocol and randomisation}

Clinical assessments included anthropometric and clinical evaluation, and sleep questionnaires. Full in-laboratory PSG was performed, and overnight urine was collected for catecholamines. Fasting blood was taken in the morning for glucose, insulin and lipids, and the short insulin tolerance test (SITT) was performed. Magnetic resonance imaging (MRI) of the abdomen was performed within 2 weeks of PSG, and before CPAP or sham CPAP treatment.

The 1-week study followed a double-blinded, randomised controlled design. Following acquisition of baseline data according to protocol, subjects were randomised to either nCPAP or sham CPAP group for 1 week of treatment. The randomisation schedule was generated by a research assistant who was independent of subject enrolment. Each randomised patient was coded numerically in a consecutive manner for analyses by the investigators at a later stage. A block randomisation was used with a block size of four, and stratified with severity. The subjects were stratified to two severity groups: AHI $>15-30$ events $\cdot \mathrm{min}^{-1}$ and $\mathrm{AHI}>30$ events $\cdot \mathrm{min}^{-1}$, to ensure that the proportions of severity groups were similar in the two treatment groups. Consecutive subjects were allocated to either of the two arms according to the randomisation schedule. Subjects were strongly advised to maintain their normal diet and exercise habits for the duration of the study. All baseline studies (except MRI abdominal fat) and PSG were repeated in both nCPAP and sham CPAP groups after 1 week, after which the treatment code was made known. Subjects in the sham CPAP group were then prescribed therapeutic nCPAP as clinically indicated, while those in nCPAP group were advised to continue treatment with a fixed pressure machine for a further 11 weeks, at the optimal pressure (95th percentile) as recorded on the autotitrating machine. Those in the nCPAP group were contacted by telephone after 6 weeks to enhance CPAP compliance, and reassessed at 12 weeks with all tests including PSG and MRI abdominal fat. After completion of the study, all study subjects were seen at the outpatient clinic (University Dept of Medicine, Queen Mary, Hospital, Hong Kong, SAR, China) for regular clinical management.

This study was approved by the Institutional Research Board Ethics Committee of the University of Hong Kong/Hong Kong Hospital Authority (West cluster), and written informed consent was obtained from all study subjects.

\section{MEASUREMENTS}

\section{Anthropometric and blood pressure measurements}

BMI was calculated from body weight and height $\left(\mathrm{kg} \cdot \mathrm{m}^{-2}\right)$. Waist circumference was measured at a level half-way between the lower rib margin and the iliac crest.

Blood pressure was measured with an appropriate cuff size in the supine position, using Dinamap (Critikon Inc., Tampa, FL, USA), in the evening at 21:00-22:00 $\mathrm{h}$ and in the morning on waking, at 07:00-08:00 $\mathrm{h}$. The average of three readings taken at 1-min intervals on each occasion was documented as evening and morning blood pressure, respectively. Morning blood pressure was used for all analyses in this study.

\section{SITT}

The SITT was conducted according to an established protocol $[21,22]$. The test was carried out at 09:00-09:30 h after a 12-h overnight fast. An indwelling catheter was inserted into an antecubital vein, with blood collection for glucose and insulin starting at $10 \mathrm{~min}$. A bolus of insulin (human actrapid; Novo Nordisk A/S, Bagsværed, Denmark) at 0.1 unit.kg-1 body weight was then administered through the catheter at time zero. Plasma glucose levels were measured at 1, 3, 5, 7, 9, 11, 13 and $15 \mathrm{~min}$ on arterialised venous samples after insulin administration. To arterialise the venous blood, the subject's hand was placed on a bag of gel beads which was held at a constant temperature of $43^{\circ} \mathrm{C}$ for $5 \mathrm{~min}$ prior to the start of the test and remained at $43^{\circ} \mathrm{C}$ until the end of the study. The test was terminated by an intravenous glucose bolus at $15 \mathrm{~min}$.

Insulin sensitivity was estimated by measuring the glucose disappearance rate, represented by the $\mathrm{K}$ constant (Kitt). Serum glucose levels were logarithmically transformed and were modelled by linear regression to estimate the slope of decline in plasma glucose concentration after insulin administration. The slope was multiplied by -100 to derive the rate constant (Kitt) which was equivalent to the percentage decline in blood glucose per minute calculated by the formula $69.3 / t_{1 / 2}$, where $t_{1 / 2}$ was the half life of the fall in plasma glucose. The coefficient of variation of Kitt was $9 \%$ as reported previously in Chinese patients [23].

\section{Homeostasis model assessment method for estimating insulin resistance}

The average insulin and glucose values of three blood samples taken over $10 \mathrm{~min}$ (at 10, 5 and $0 \mathrm{~min}$ before SITT) were used 
for calculating the homeostasis model assessment method for estimating insulin resistance (HOMA-IR): fasting plasma glucose $\left(\mathrm{mmol} \cdot \mathrm{L}^{-1}\right) \times$ fasting serum insulin $\left(\mathrm{mIU} \cdot \mathrm{L}^{-1}\right) / 22.5$ [24]. For more information refer to the online supplement.

\section{Lipid profile}

Plasma total cholesterol and triglyceride were determined enzymatically on a Hitachi 912 analyser (Roche Diagnostics, $\mathrm{GmbH}$, Mannheim, Germany). Apolipoprotein B was measured by rate nephelometry using the Beckman Array System (Beckman Instruments, Fullerton, CA, USA). For more information refer to the online supplement.

\section{Urinary catecholamines}

Urine was collected between 22:00 $\mathrm{h}$ and 08:00 $\mathrm{h}$ on the night of sleep study for assays of catecholamines and their metabolites (epinephrine, norepinephrine, metanephrine and normetanephrine). For more information refer to the online supplement.

\section{MRI for intra-abdominal fat}

Subjects underwent abdominal MRI using a $1.5 \mathrm{~T}$ magnet (Signa Horizon LX; General Electric Medical Systems, Milwaukee, WI, USA) in the morning, having fasted for $\geqslant 4 \mathrm{~h}$. For more information refer to the online supplement.

\section{Statistical analysis}

The primary end-point was the difference in the change in Kitt after 1 week of therapeutic or sham nCPAP treatment. The intention-to-treat principle was applied in the analyses between treatment groups, but missing data were not imputed to avoid dilution effect. Within-group comparisons were examined by a paired t-test for normally distributed data or Wilcoxon signed rank test for non-normally distributed data. Between-group comparisons were examined by an independent sample unpaired t-test for normally distributed data or the Mann-Whitney U-test for non-normally distributed data. The changes over 12 weeks in the nCPAP group were all analysed by Wilcoxon signed rank tests due to small sample size. Stepwise forward regression analyses were performed to identify predictors for the changes in Kitt at 1 week using patients in both groups, and at 12 weeks using patients in the nCPAP group. The selected models were re-fitted to all patients with available data in the selected predictors to obtain final estimates of the regression coefficients. Additional subset analysis was performed for the changes in Kitt or other variables by BMI groups with Wilcoxon signed ranks. All statistical tests were two-sided and the p-values for the secondary analyses were not adjusted for multiple tests due to the exploratory nature. Analysis was performed with SPSS version 15.0 (SPSS Inc., Chicago, IL, USA).

\section{Sample size calculation}

Our pilot work on changes of Kitt in five subjects who received nCPAP treatment for 5-7 days estimated the mean \pm SD change of Kitt as $1.7 \pm 1.5 \mathrm{mmol} \cdot \mathrm{L}^{-1} \cdot \mathrm{min}^{-1}$. Assuming a common SD of $1.5 \mathrm{mmol} \cdot \mathrm{L}^{-1} \cdot \mathrm{min}^{-1}$ for both groups and a difference in change of $1.5 \mathrm{mmol} \cdot \mathrm{L}^{-1} \cdot \mathrm{min}^{-1}$ between the two groups, at a significance level of $5 \%$ and with a power of $90 \%$, we required a minimum of 21 subjects in each group. Allowing for spontaneous dropouts, defaults and poor nCPAP adherence, 30 subjects per group were targeted.

\section{RESULTS}

Figure 1 shows the flowchart of the study. In total, 964 diagnostic PSGs were performed during the study period, only $70(7.3 \%)$ subjects were eligible for the study. However, one patient had been recently diagnosed with familial hypertriglyceridemia, three had been diagnosed with diabetes mellitus after having baseline blood tests and five patients refused to participate. Thus, 61 subjects who fulfilled the criteria were recruited, with a mean \pm SD age of $46.3 \pm 10.2$ yrs, BMI of $27.5 \pm 3.7 \mathrm{~kg} \cdot \mathrm{m}^{-2}$ and AHI of $39.7 \pm 22.1$ events $\cdot \mathrm{h}^{-1}$.

The patients were randomised to either the nCPAP $(n=31)$ or sham CPAP $(n=30)$ group for 1 week of treatment. In the nCPAP group, one subject did not return for reassessment at 1 week but at 2 weeks, one SITT failed technically and several samples of urinary catecholamines had assay interference (table 1). In the sham CPAP group, one subject did not use the treatment device at all during the study period, one subject modified the CPAP machine to block the extra holes in the exhalation port of the mask, one SITT failed technically and several samples of urinary catecholamines had assay interference (table 1). All subjects were included and analysed in the groups that they were first assigned to, with the exception of failed SITT and urinary assay interference where no data were available for analyses.

At baseline, the two groups had no significant difference in Kitt and other parameters (table 1). 1 week CPAP use was higher in the nCPAP group than the sham CPAP group $\left(6.2 \pm 1.5 \mathrm{~h} \cdot\right.$ night $^{-1}$ versus $4.5 \pm 2.0 \mathrm{~h} \cdot$ night $\left.^{-1} ; \mathrm{p}=0.001\right)$.

After 1 week of therapeutic nCPAP treatment, the group had a significant increase in Kitt $(p=0.017)$ and decrease in systolic and diastolic blood pressure $(p=0.008$ and $p=0.004$, respectively), while the sham CPAP group, which also had a significant but much smaller reduction in AHI, showed no significant change in Kitt or systolic blood pressure but a decrease in diastolic blood pressure $(\mathrm{p}=0.021)$ (table 1, fig. 2a). Between group comparisons showed that the changes in Kitt with treatment were significantly different $(p=0.022)$ (table 1$)$. All subjects reported no changes in diet and physical activity. Further subgroup analysis within the nCPAP group according to $\mathrm{BMI}<25$ and $\geqslant 25 \mathrm{~kg} \cdot \mathrm{m}^{-2}$, the Asian criteria for obesity [25], was conducted and improvement was only seen in the 22 patients with a BMI $\geqslant 25 \mathrm{~kg} \cdot \mathrm{m}^{-2}$ (median (range) 28.3 $(26.6,31.7) \mathrm{kg} \cdot \mathrm{m}^{-2}$, Kitt $\mathrm{p}=0.002$, systolic blood pressure $p=0.007$ and diastolic blood pressure $p=0.011$ ), but not in the nine patients with a BMI $<25 \mathrm{~kg} \cdot \mathrm{m}^{-2}\left(24.5(24.1,25) \mathrm{kg} \cdot \mathrm{m}^{-2}\right)$ with a significant difference for between-group change in Kitt $(p=0.022)$. In the stepwise forward regression analysis, the only significant predictor for the changes in Kitt after 1 week of treatment was urinary normetanephrine (table 2).

After 12 weeks of therapeutic nCPAP treatment, 29 subjects were reassessed as two refused to return to the outpatient clinic for reassessment. Mean nCPAP use during 12 weeks of treatment was $4.9 \pm 1.4 \mathrm{~h} \cdot$ night $^{-1}$. No significant changes occurred in BMI, waist circumference and total abdominal fat on MRI over the study period (table 3 ). In the group using CPAP for 12 weeks, Kitt showed no significant change 
compared with baseline, while there were significant reductions in systolic and diastolic blood pressure, total cholesterol, triglycerides, apolipoprotein B, and urinary norepinephrine and epinephrine (table 3). On further subgroup analysis for obesity (BMI $<25 \mathrm{~kg} \cdot \mathrm{m}^{-2}, \mathrm{n}=9$; BMI $\geqslant 25 \mathrm{~kg} \cdot \mathrm{m}^{-2}, \mathrm{n}=20$ ), Kitt was significantly improved in obese subjects $(p=0.044)$ (fig. 2b), but not in nonobese subjects, and the between-group comparison of changes was also significantly different $(\mathrm{p}=0.009)$. In the multiple regression analysis (table 2), BMI $<25 / \geqslant 25 \mathrm{~kg} \cdot \mathrm{m}^{-2}$ was the only independent predictor for the changes in Kitt at 12 weeks. Other parameters including systolic and diastolic blood pressure, total cholesterol, triglycerides, apolipoprotein B, urinary norepinephrine and epinephrine also showed significant changes in the obese group but not the nonobese group.

\section{DISCUSSION}

In this short-term, randomised controlled study in otherwise healthy males with moderate-to-severe OSA, we were able to demonstrate that 1 week of effective nCPAP treatment significantly increased insulin sensitivity. This improvement was only maintained in those who were obese in the open treatment group over 12 weeks. These findings suggest that OSA is an independent risk factor for adverse glucose metabolism, but the detrimental effects may be less prominent in nonobese subjects compared with moderately obese subjects. The pathogenetic pathway of impaired insulin sensitivity may involve sympathetic activation and other adiposity-related mechanisms.

A number of cross-sectional studies have reported that OSA is independently associated with insulin resistance/glucose intolerance, suggesting that OSA may play a causative role in the development of type 2 diabetes and the metabolic syndrome, while some other studies did not identify any independent association after adjusting for obesity [6]. Despite more prevalent diabetes in OSA subjects at baseline, no independent relationship could be found between OSA and incident diabetes at 4-yr follow-up [7].

An effect of intervention of one condition on another is regarded as strong evidence for a causal link. Previous studies of the effects of nCPAP treatment of OSA on insulin resistance or glycemic control have reported variable results. Many failed to show any improvement in glucose metabolism [6]. Using the hyperinsulinemic euglycemic clamp, two nights of nCPAP treatment was reported to improve insulin sensitivity in a group of nondiabetic Caucasian males with moderate OSA [10], and the improvement was maintained at 3 yrs [26]. However, in a randomised controlled study with a cross-over design, no change in HOMA-IR was found in Caucasian males with severe OSA after 6 weeks of CPAP treatment compared with sham-CPAP [13]. Recently, a case-control study showed

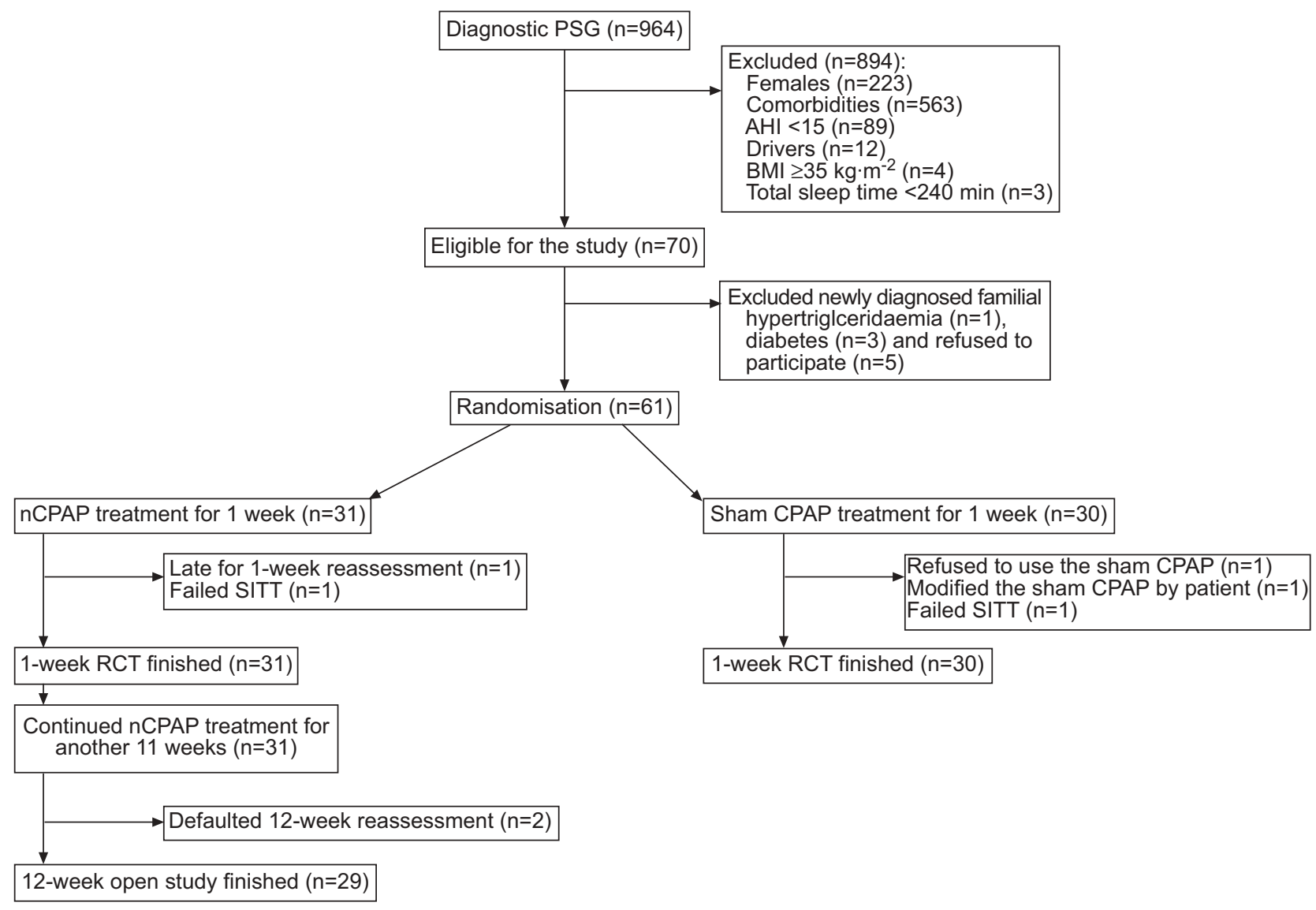

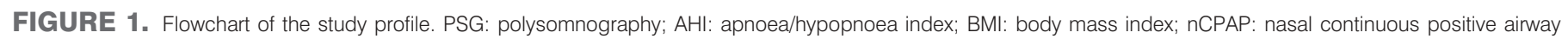
pressure; CPAP: continuous positive airway pressure; SITT: short insulin tolerance test; RCT; randomised controlled trial. 


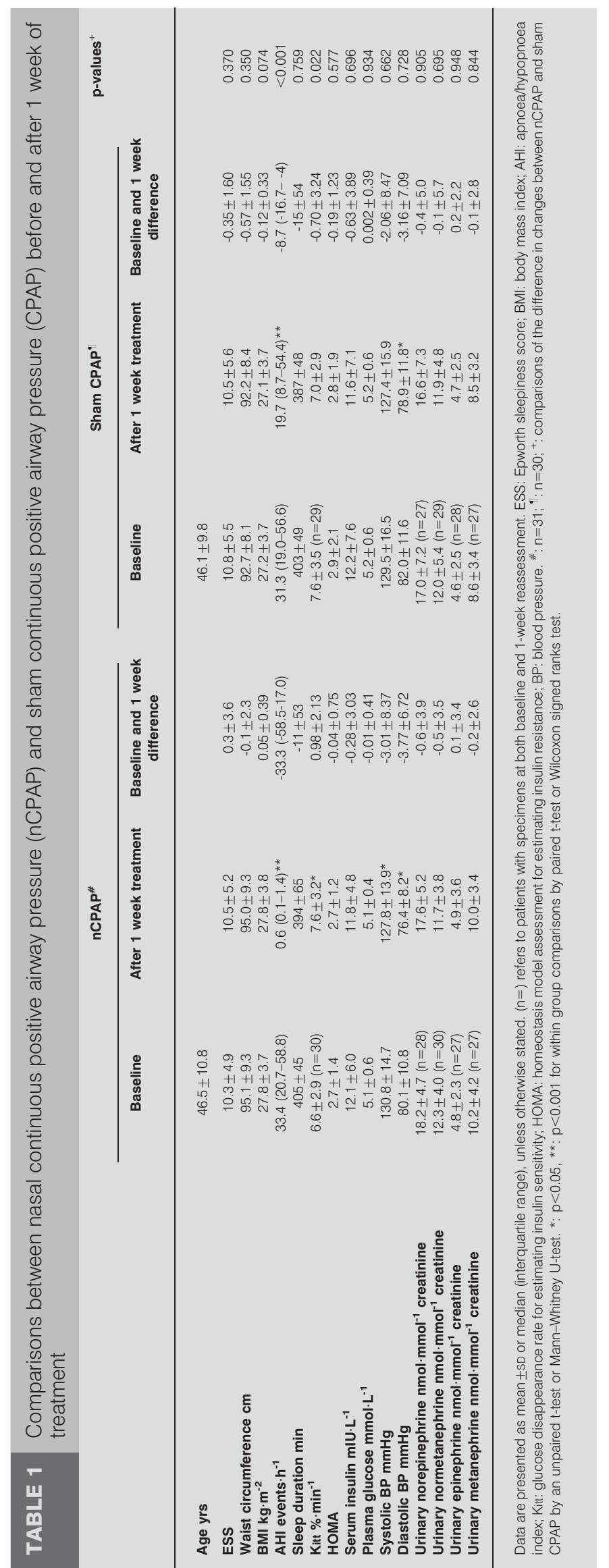

that 3 months of nCPAP treatment reduced HOMA-IR in sleepy OSA subjects but not in nonsleepy subjects [16]. Observational studies further reported that CPAP compliance determined a positive outcome in HOMA-IR [15], or a decrease in glycosylated haemoglobin but not HOMA-IR [17]. Data on changes in fasting insulin or HOMA-IR in children before and after adenotonsillectomy were similarly variable [27, 28]. In diabetic subjects with OSA, despite several positive observational studies $[6,8,11,12]$, a randomised controlled study reported that neither $\mathrm{HbA}_{1 \mathrm{C}}$ nor insulin sensitivity measured by the euglycemic clamp changed [14].

It is obvious that different methods have been used in the evaluation of in vivo glucose metabolism [6], ranging from simple measurements of fasting blood glucose and insulin to the sophisticated hyperinsulinemic euglycemic clamp study, which is considered as the "gold standard" for measuring insulin sensitivity [29]. The limitations and strengths of each of these methods cast significant impact on the interpretation of their results [21, 24, 29]. In our study, the primary outcome measure was obtained by the SITT, a test for insulin sensitivity which has been validated against clamp studies [21] and been shown to be reasonably reproducible [22]. It is a relatively rapid and simple test in which an estimate of insulin sensitivity is obtained from the rate of decline in glucose levels following an intravenous bolus of insulin. The test reflects the combination of suppression of hepatic glucose output and stimulation of peripheral glucose uptake by insulin, representing peripheral insulin resistance [29]. The short duration of the test avoids the problem of interference from the release of counterregulatory hormones [29]. Compared with Kitt, HOMA-IR is a mathematical modelling of basal plasma insulin and glucose which predominantly expresses the ability of basal insulin to suppress hepatic glucose production in a fasting state. The test represents hepatic insulin resistance and is subject to variability in $\beta$-cell function in a feed-back loop [24]. Catecholamines can inhibit insulin secretion by activating $\alpha_{2^{-}}$ adrenoreceptors in $\beta$-cells [30]. Exposure to intermittent hypoxia in young healthy adults was shown to impair insulin sensitivity with a lack of compensatory hyperinsulinemia, suggesting a concomitant suppression of $\beta$-cell function [31]. Similarly using the intravenous glucose tolerance test, OSA subjects were also found to have impairment in insulin sensitivity, $\beta$-cell function and glucose effectiveness [32]. It is logical to surmise that effective treatment of OSA may also improve $\beta$-cell function with increased insulin secretion. With these intrinsic limitations, the HOMA-IR may underestimate any improvement in insulin sensitivity, and remains unchanged when Kitt improves.

The exact mechanisms by which OSA leads to insulin resistance are not fully understood. One potential mediating mechanism is the elevation of sympathetic activity [33]. Our regression analysis for predictors of changes in insulin sensitivity at 1 week supported this postulated mechanism, albeit the regression model indicated that it only accounted for a small degree of the variance.

The effect of co-existent obesity on glucose metabolism in OSA has been a contentious issue. Obesity, especially visceral obesity, is an important determinant of insulin sensitivity, and an inevitable confounder in studies of adverse metabolic 

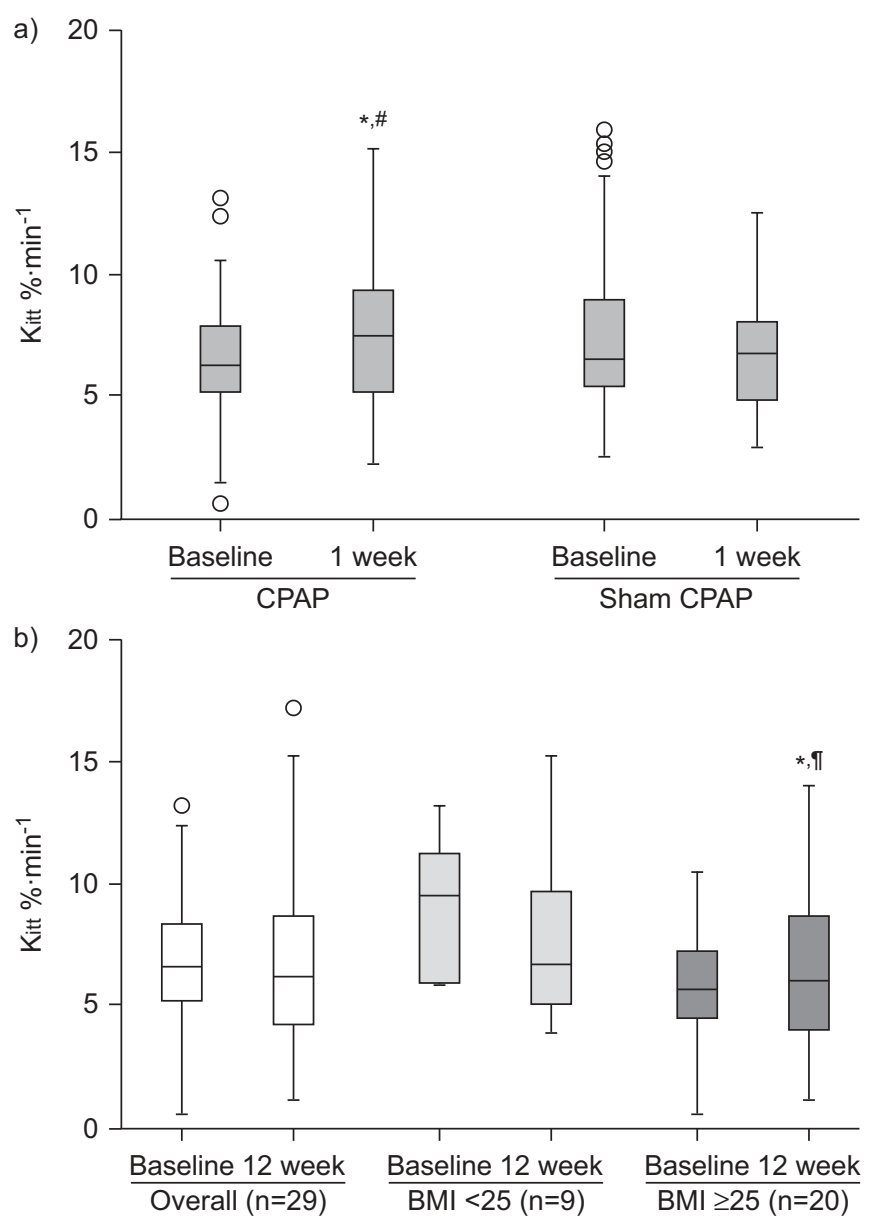

FIGURE 2. a) Changes in the glucose disappearance rate for estimating insulin sensitivity (Kitt) at baseline and after 1 week of continuous positive airway pressure (CPAP) and sham CPAP treatments. b) Changes in Kitt after 12 weeks of CPAP treatment overall $\left(\square: \mathrm{n}=29\right.$ ), with body mass index $(\mathrm{BMI})<25 \mathrm{~kg} \cdot \mathrm{m}^{-2}$ ( $\square: n=9)$ and $B M I \geqslant 25 \mathrm{~kg} \cdot \mathrm{m}^{-2}(\square: \mathrm{n}=20) .{ }^{*}: \mathrm{p}<0.05$ for comparisons of the changes between groups; ${ }^{\uparrow}: p<0.05$ for comparisons of the changes between $\mathrm{BMl}<25$ and $\mathrm{BMI} \geqslant 25$. ${ }^{*}: \mathrm{p}<0.05$ for within group comparison.

effects of OSA. Previous studies suggested that both obese and nonobese subjects had adverse glucose metabolism related to OSA [34, 35]. However, insulin sensitivity in response to nCPAP treatment was found to be better in nonobese Caucasian males (BMI $<30 \mathrm{~kg} \cdot \mathrm{m}^{-2}$ ) compared to those with
$\mathrm{BMI} \geqslant 30 \mathrm{~kg} \cdot \mathrm{m}^{-2}[10]$. In contrast, a paediatric study suggested that OSA played a more significant role in insulin sensitivity in obese compared with nonobese children [27]. We have excluded, by design, those who were morbidly obese, with the speculation that severe obesity may have an overwhelming effect on insulin resistance masking any contribution by OSA. Our data showed no changes of BMI, waist circumference and the volume of visceral fat after 12 weeks of nCPAP treatment, and moderately obese subjects had a better metabolic response compared with the nonobese. MRI abdomen might not be the best tool to estimate the body fat content as it does not reflect total body fat mass. Nevertheless, we have used various indicators of body habitus and body fat, and showed no significant change in adiposity. Although the physical mass is static, the composites of adiposity might have changed at the molecular levels after treatment. A number of inflammatory and neurohumoral mediators are produced from the adipose tissues, especially from the visceral adipose tissues which have been demonstrated to be involved in the pathogenesis of insulin resistance $[33,36]$. With the application of nCPAP treatment in the obese apnoeic group, the changes of these mediators and, hence, insulin sensitivity might have been more pronounced than the nonobese apnoeic group.

Apart from the randomised controlled design, and the use of a more precise measurement of insulin sensitivity, other strengths of our study include the satisfactory CPAP compliance and the inclusion of only subjects with no significant comorbidity to allow clear delineation of the impact of OSA per se on glucose metabolism. Our findings strongly support the notion that OSA has a causal role on insulin resistance, and the improvement with CPAP treatment carries clinical relevance, as a similar improvement in Kitt has been shown after weight reduction by different methods $[37,38]$. In a prospective study of 31 morbidly obese females with a reduction of mean BMI from 54 to $35 \mathrm{~kg} \cdot \mathrm{m}^{-2} 1 \mathrm{yr}$ post-bariatric surgery, Kitt was improved by $1-3 \%$ in groups of different glucose profiles [37]. In a randomised placebo-controlled study of sibutramineassisted weight reduction of $5.6 \mathrm{~kg}$ in nondiabetic subjects, mean Kitt was enhanced significantly from $4-5 \% \cdot \mathrm{min}^{-1}$ in the treatment group compared with no changes in the control group which had no weight reduction [38].

However, the sample characteristics by design also mean that our results may not be extrapolated to females, those with mild-to-moderate OSA, and those with comorbidities including diabetes who form a large proportion of sleep clinic

TABLE 2 Forward stepwise regression models for the changes in insulin sensitivity

\section{Adjusted $\mathbf{R}^{2}$}

$7.4 \%$

$22.8 \%$
Estimate (SE)

$-0.184(0.078)$

$2.928(0.962)$ p-value

0.023

0.005

After 12 weeks of nCPAP treatment

$\mathrm{BMl}<25 / \mathrm{BMl} \geqslant 25 \mathrm{~kg} \cdot \mathrm{m}^{-2}$

Independent variables considered were: age, waist circumference, body mass index (BMI) $<25 / \mathrm{BMl} \geqslant 25 \mathrm{~kg} \cdot \mathrm{m}^{-2}$, sleep parameters at baseline (apnoea/hypopnoea index, arousal index, duration with $\mathrm{O}_{2}$ saturation $<90 \%$ and minimum $\mathrm{O}_{2}$ saturation), changes in urinary norepinephrine, normetanephrine, epinephrine and metanephrine. nCPAP: nasal continuous positive airway pressure. ${ }^{*}: n=57 ;{ }^{\circ}: n=29$. 
TABLE 3 Before and after 12 weeks of nasal continuous positive airway pressure treatment in 29 subjects

\begin{tabular}{|c|c|c|c|}
\hline & Baseline & After 12 weeks & p-value ${ }^{\#}$ \\
\hline AHI events $\cdot h^{-1}$ & $36.8(19.0-59.3)$ & $0.6(0-1.1)$ & $<0.001$ \\
\hline Waist circumference $\mathrm{cm}$ & $94(89-100)$ & $95(88.3-100.0)$ & 0.966 \\
\hline BMI $\mathbf{k g} \cdot \mathrm{m}^{-2}$ & $26.7(25.0-30.3)$ & $26.8(25.4-29.8)$ & 0.421 \\
\hline MRI visceral fat $\mathrm{cm}^{3}$ & $1113.9(730.1-1431.3)$ & $1002.4(870.2-1270.7)$ & 0.407 \\
\hline НОМА & $2.5(1.5-3.4)$ & $2.1(1.5-3.4)$ & 0.511 \\
\hline Serum insulin $\mathrm{mIU} \cdot \mathrm{L}^{-1}$ & $10.9(7.3-14.0)$ & $10.7(7.1-15.4)$ & 0.199 \\
\hline Plasma glucose $\mathrm{mmol} \cdot \mathrm{L}^{-1}$ & $4.9(4.7-5.4)$ & $5.1(4.7-5.3)$ & 0.954 \\
\hline Systolic BP $\mathrm{mmHg}$ & $132(122-141)$ & $123(117-134)$ & 0.021 \\
\hline Diastolic BP $\mathrm{mmHg}$ & $81(73-88)$ & $76(71-82)$ & 0.042 \\
\hline Total cholesterol $\mathrm{mmol} \cdot \mathrm{L}^{-1}$ & $5.1(4.8-5.7)$ & $4.8(4.2-5.6)$ & 0.016 \\
\hline Apolipoprotein $\mathrm{B} \mathrm{mmol} \cdot \mathrm{L}^{-1}$ & $1.1(0.9-1.2)$ & $1.0(0.8-1.1)$ & 0.003 \\
\hline Urinary norepinephrine $\mathrm{nmol} \cdot \mathrm{mmol}^{-1} \mathrm{creatinine}$ & $19.0(15.3-22.0)(n=22)$ & $15.5(11.0-17.8)$ & 0.022 \\
\hline Urinary normetanephrine $\mathrm{nmol} \cdot \mathrm{mmol}^{-1}$ creatinine & $12.0(9.4-15.0)(n=27)$ & $11(7.5-13.0)$ & 0.063 \\
\hline Urinary epinephrine $\mathrm{nmol} \cdot \mathrm{mmol}^{-1} \mathrm{creatinine}$ & $4.1(3.4-6.1)(n=22)$ & $3.6(1.95 .2)$ & 0.027 \\
\hline Urinary metanephrine $\mathrm{nmol} \cdot \mathrm{mmol}^{-1} \mathrm{creatinine}$ & $9.5(7.0-12.0)(n=26)$ & $9.3(6.8-12.3)$ & 0.399 \\
\hline
\end{tabular}

patients. A previous study has suggested that nCPAP treatment only improved insulin resistance, as reflected by HOMA-IR, in sleepy but not nonsleepy OSA subjects [16]. On average, our study subjects had only mild sleepiness, and the sample size was not adequate for further sub-group analysis for any influence of sleepiness on the response in insulin sensitivity. For further delineation of the impact of OSA on glucose metabolism, it is necessary to evaluate the effect of nCPAP on $\beta$-cell function [39] and noninsulin dependent mechanisms, and the interaction of OSA and adiposity. Finally, we only studied the pathogenetic role of sympathetic activation in insulin resistance, while many other potential mechanisms, such as inflammation, have not been evaluated.

In conclusion, the findings of our study demonstrate that treatment of OSA improved insulin sensitivity in the short term, suggesting a causal relationship between OSA and insulin resistance. The effect appeared to be maintained in the moderately obese subjects over 12 weeks. Early effective nCPAP treatment of OSA may alter the natural course of glucose metabolism favourably in otherwise healthy subjects.

\section{SUPPORT STATEMENT}

This study was supported by Lee Wing Tat Cardiorespiratory Research Fund, The University of Hong Kong (Hong Kong, SAR, China).

\section{STATEMENT OF INTEREST}

A statement of interest for M. Ip can be found at www.erj.ersjournals. $\mathrm{com} / \mathrm{misc} /$ statements.dtl

\section{ACKNOWLEDGEMENTS}

We would like to thank to P.P. Ku and J. Lam (both Division of Respiratory and Critical Care Medicine, Queen Mary Hospital, The University of Hong Kong, Hong Kong), for their technical support in the manual scoring of all polysomnography performed in this study.

\section{REFERENCES}

1 McNicholas WT, Bonsignore MR, Management Committee of EU COST ACTION B26Sleep apnoea as an independent risk factor for cardiovascular disease: current evidence, basic mechanisms and research priorities. Eur Respir J 2007; 29: 156-178.

2 Marin JM, Carrizo SJ, Vicente E, et al. Long-term cardiovascular outcomes in men with obstructive sleep apnoea-hypopnoea with or without treatment with continuous positive airway pressure: an observational study. Lancet 2005; 365: 1046-1053.

3 Gami AS, Somers VK. Obstructive sleep apnea, metabolic syndrome, and cardiovascular outcomes. Eur Heart J 2004; 25: 709-711.

4 de Sousa AGP, Cercato C, Mancini MC, et al. Obesity and obstructive sleep apnea. Obes Rev 2008; 9: 340-354.

5 Consensus development conference on insulin resistance. 5-6 November 1997. American Diabetes Association. Diabetes Care 1998; 21: 310-314.

6 Tasali E, Ip MSM. Obstructive sleep apnea and metabolic syndrome - alterations in glucose metabolism and inflammation. Proc Am Thorac Soc 2008; 5: 207-217.

7 Reichmuth KJ, Austin D, Skatrud JB, et al. Association of sleep apnea and type II diabetes: a population-based study. Am J Respir Crit Care Med 2005; 172: 1590-1595. 
8 Brooks B, Cistulli PA, Borkman M, et al. Obstructive sleep apnea in obese noninsulin-dependent diabetic patients: effect of continuous positive airway pressure treatment on insulin responsiveness. $J$ Clin Endocrinol Metab 1994; 79: 1681-1685.

9 Smurra M, Philip P, Taillard J, et al. CPAP treatment does not affect glucose-insulin metabolism in sleep apneic patient. Sleep Med 2001; 2: 207-213.

10 Harsch IA, Schahin SP, Radespiel-Troger M, et al. Continuous positive airway pressure treatment rapidly improves insulin sensitivity in patients with obstructive sleep apnea syndrome. Am J Respire Crit care Med 2004; 169: 156-162.

11 Babu AR, Herdegen J, Fogelfeld L, et al. Type 2 diabetes, glycemic control, and continuous positive airway pressure in obstructive sleep apnea. Arch Intern Med 2005; 165: 447-452.

12 Hassaballa HA, Tulaimat A, Herdegen JJ, et al. The effect of continuous positive airway pressure on glucose control in diabetic patients with severe obstructive sleep apnea. Sleep Breath 2005; 9: 176-180.

13 Coughlin SR, Mawdsley L, Mugarza JA, et al. Cardiovascular and metabolic effects of CPAP in obese males with OSA. Eur Respir J 2007; 29: 720-727.

14 West SD, Nicoll DJ, Wallace TM, et al. The effect of CPAP on insulin resistance and $\mathrm{HbA1C}$ in men with obstructive sleep apnoea and type 2 diabetes. Thorax 2007; 62: 969-974.

15 Dorkova Z, Petrasova D, Molcanyiova A, et al. Effects of CPAP on cardiovascular risk profile in patients with severe obstructive sleep apnea and metabolic syndrome. Chest 2008; 134: 686-692.

16 Barcelo A, Barbe F, de la Pena M, et al. Insulin resistance and daytime sleepiness in patients with sleep apnoea. Thorax 2008; 63: 946-950.

17 Steiropoulos P, Papanas N, Nena E, et al. Markers of glycemic control and insulin resistance in non-diabetic patients with obstructive sleep apnea hypopnea syndrome: does adherence to CPAP treatment improve glycemic control? Sleep Med 2009; 10: 887-891.

18 Lam JCM, Xu A, Tam S, et al. Hypoadiponectinemia is related to sympathetic activation and severity of obstructive sleep apnea. Sleep 2008; 31: 1721-1727.

19 Farre R, Hermamdez L, Montserrat JM, et al. Sham continuous positive airway pressure for placebo-controlled studies in sleep apnoea. Lancet 1999; 353: 1154.

20 Pepperell JCT, Ramdassingh-Dow S, Crosthwaite $\mathrm{N}$, et al. Ambulatory blood pressure after therapeutic and subtherapeutic nasal continuous positive airway pressure for obstructive sleep apnoea: a randomized parallel trial. Lancet 2001; 359: 204-210.

21 Bonora E, Moghetti P, Zancanaro C, et al. Estimates of in vivo insulin action in man: comparison of insulin tolerance tests with euglycemic and hyperglycaemic glucose clamp studies. J Clin Endocrinol Metab 1989; 68: 374.

22 Hirst S, Philips DIW, Vines SK, et al. Reproducibility of the short insulin tolerance test. Diab Med 1993; 10: 839-842.
23 Tan KCB, Mackay IR, Zimmet PZ, et al. Metabolic and immunologic features of Chinese patients with atypical diabetes mellitus. Diabetes Care 2000; 23: 335-338.

24 Matthews DR, Hosker JP, Rudenski AS, et al. Homeostasis model assessment: insulin resistance and beta cell function from fasting plasma glucose and insulin concentrations in man. Diabetologia 1985; 28: 412-419.

25 WHO expert consultationAppropriate body-mass index for Asian populations and its implications for policy and intervention strategies. Lancet 2004; 363: 157-163.

26 Schahin SP, Nechanitzky T, Dittel C, et al. Long-term improvement of insulin sensitivity during CPAP therapy in the obstructive sleep apnoea syndrome. Med Sci Monit 2008; 14: CR117-CR121.

27 Gozal D, Sans Capdevila O, Kheirandish-Gozal L. Metabolic alterations and systemic inflammation in obstructive sleep apnea among nonobese and obese prepubertal children. Am J Respir Crit Care Med 2008; 177: 1142-1149.

28 Verhulst SL, Rooman RP, Van Gaal L, et al. Is sleep-disordered breathing an additional risk factor for the metabolic syndrome in obese children and adolescents? Int J Obes (Lond) 2009; 33: 8-13.

29 Wallace TM, Matthews DR. The assessment of insulin resistance in man. Diabet Med 2002; 19: 527-534.

30 Chan SLF, Pernett CW, Morgan NG. Differential expression of alpha $_{2}$-adrenoceptor subtypes in purified rat pancreatic islet Aand B-cells. Cell signal 1997; 9: 71-78.

31 Louis M, Punjabi NM. Effects of acute intermittent hypoxia on glucose metabolism in awake healthy volunteers. J Appl Physiol 2009; 106: 1538-1544.

32 Punjabi NM, Beamer BA. Alterations in glucose disposal in sleepdisordered breathing. Am J Respir Crit Care Med 2008; 179: 235-240.

33 Trakada G, Chrousos G, Pejovic S, et al. Sleep apnea and its association with the stress system, inflammation, insulin resistance and visceral obesity. Sleep Med Clin 2007; 2: 251-261.

34 Ip MSM, Lam B, Ng MMT, et al. Obstructive sleep apnoea is independently associated with insulin resistance. Am J Respir Crit Care Med 2002; 165: 670-676.

35 Seicean S, Kirchner HL, Gottlieb DJ, et al. Sleep-disordered breathing and impaired glucose metabolism in normal-weight and overweight/obese individuals. Diab Care 2008; 1001-1006.

36 Lam DCL, Xu A, Lam KSL, et al. Serum adipocyte-fatty acid binding protein (A-FABP) level is elevated in severe obstructive sleep apnea (OSA) and correlates with insulin resistance. Eur Respir J 2009; 33: 346-351.

37 Geloneze B, Tambascia MA, Pareja JC, et al. The insulin tolerance test in morbidly obese patients undergoing bariatric surgery. Obes Res 2001; 9: 763-769.

38 Tambascia MA, Geloneze B, Repetto EM, et al. Sibutramine enhances insulin sensitivity ameliorating metabolic parameters in a double-blind, randomized, placebo-controlled trial. Diabetes Obes Metab 2003; 5: 338-344.

39 Kahn SE. The importance of the beta-cell in the pathogenesis of type 2 diabetes mellitus. Am J Med 2000; 109: Suppl. 6a, 2S-8S. 